\title{
SURFACE MORPHOLOGY OF POLYIMIDE THIN FILM DIP-COATED ON POLYESTER FILAMENT FOR DIELECTRIC LAYER IN FIBROUS ORGANIC FIELD EFFECT TRANSISTOR
}

\author{
Lina Rambausek ${ }^{1}$, Els Bruneel ${ }^{2}$, Isabel Van Driessche ${ }^{3}$, Lieva Van Langenhove ${ }^{4}$ \\ ${ }^{1}$ Ghent University - Department of Textiles, Ghent, Belgium, Technologiepark 907, 9052 Zwijnaarde, \\ tel +32 926454 06, fax +32 926458 31, Lina.Rambausek@UGent.be \\ ${ }^{2}$ Ghent University - Department of Inorganic and Physical Chemistry, Ghent, Belgium, Krijgslaan 281 S3, 9000 Ghent, \\ tel. +32 926444 47, fax +32 926449 83, Els.Bruneel@UGent.be \\ ${ }^{3}$ Ghent University - Department of Inorganic and Physical Chemistry, Ghent, Belgium, Krijgslaan 281 S3, 9000 Ghent, \\ tel. +32 926444 47, fax +32 926449 83, Isabel.VanDriessche@UGent.be \\ ${ }^{4}$ Ghent University - Department of Textiles, Ghent, Belgium, Technologiepark 907, 9052 Zwijnaarde, \\ tel +32 926454 06, fax +32 926458 31, Lieva.VanLangenhove@UGent.be
}

\begin{abstract}
:
The idea of wearable electronics automatically leads to the concept of integrating electronic functions on textile substrates. Since this substrate type implies certain challenges in comparison with their rigid electronic companions, it is of utmost importance to investigate the application of materials for generating the electronic functions on the textile substrate. Only when interaction of materials and textile substrate is fully understood, the electronic function can be generated on the textile without changing the textile's properties, being flexible or stretchable. This research deals with the optimization of the dielectric layer in a fibrous organic field effect transistor (OFET). A transistor can act as an electrical switch in a circuit. In this work, the polyimide layer was dip-coated on a copper-coated polyester filament. After thoroughly investigating the process conditions, best results with minimal thickness and roughness at full insulation could be achieved at a dip-coating speed of $50 \mathrm{~mm} / \mathrm{min}$. The polyimide solution was optimal at $15 \mathrm{w} \%$ and the choice for the solvent NMP was made. In this paper, details on the pre-treatment methods, choice of solvent and dip-coating speed and their effect on layer morphology and thickness, electrical properties and roughness are reported. Results show that the use of polyimide as a dielectric layer in the architecture of a fibrous OFET is promising. Further research deals with the application of the semiconductor layer within the mentioned architecture, to finally build an OFET on a filament for application in smart textiles.
\end{abstract}

\section{Keywords:}

Polyimide, dielectric, organic field effect transistor, filament, dip-coating

\section{Introduction}

The area of smart textiles is highly multidisciplinary and, therefore, includes aspects from the scientific terrains of textiles and electronics. Material science as well as physics and chemistry have an undeniable impact. Besides academia, also industry research strives for making truly integrated textile electronics [1-3].

True integration points at a textile substrate incorporating an electronic function, invisibly integrated and unrecognizable to touch. To achieve this level of integration, the textile substrate itself needs to act as an electrical component and should independently fulfil the electronic function of, e.g. a resistor, capacitor or transistor. To manufacture such components on textile substrates, deposition of multiple layers with different functionalities is required. In this respect, manufacturing techniques from the electronics industry need to be adapted to the textile substrate's versatile properties, such as flexibility or elasticity [4-7] and surfaces with curvature. Many filaments are of cylindrical shape, if materials are deposited onto this threedimensional body, they respond to the curvature in different ways regarding, e.g. adhesion or crystallization [8]. It is essential to know how to transfer materials from the electronic world onto textile substrates to generate a basic electronic function. For the transistor, this would be an electrical switch or a sensor, for instance. The understanding will serve as the fundament for further developing sophisticated smart textile products, up to the futuristic concept of the wearable computer.

This systematic study is directed towards greater understanding of the fundamental aspects in coating of polyester (PES) filaments with a variety of materials, in view of developing an organic field effect transistor (OFET). To achieve this, a topcontact-bottom-gate (TCBG) layered architecture (Figure 1) is applied onto the filament using different consecutive coating techniques.

The conductive gate layer is obtained by electroless deposition of copper on the polyester filament, which has been discussed extensively in previous publications $[3,9]$. Subsequently, onto the copper-coated PES filament, the dielectric and the semiconductive layer are applied successively by dipcoating. To complete the architecture, the conductive top contacts, source and drain are added, e.g. by evaporation. 


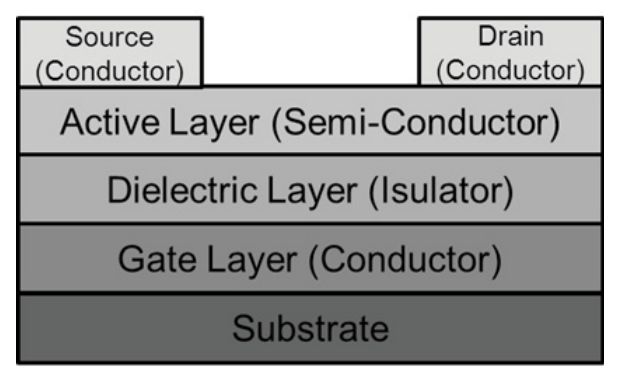

Figure 1. Schematic view of OFET Top-Contact-Bottom-Gate (TCBG) architecture

Until now, dip-coating of the dielectric layer with polyimide has only been tested on metal copper wires [10] and polyester ribbons [3] but not on copper-coated polyester filaments. One of the reasons for this might be that realization of reliable conductive filaments, coated by electroless copper plating, has not been achieved in the past [3]. Nowadays, we are able to successfully reproduce conductive copper-coated polyester filaments with a diameter of $888 \mu \mathrm{m}$ [2], which enables us to further engage with this research. Using a copper-coated PES filament in comparison to a copper wire has the advantage that the filament is not reluctant to break, it is flexible and therefore is the better choice when thinking about, e.g. intelligent clothing [11].

For optimal transistor properties, a homogeneous insulating layer with constant minimum thickness and low roughness is a prerequisite [12-14]. Existing micro-voids in the layer are likely to act as traps for the charge carriers and will have a negative effect on field effect mobility and the overall transistor performance. The quality of the dielectric layer was evaluated by determining its insulating properties through resistivity and leak current measurements. The insulating properties were demonstrated by Maccioni et al. who used a cylindrical metal fibre with a uniform polyimide layer of $1 \mu \mathrm{m}$ (purchased from Elektrisola) indicating a leak current of below $10^{10} \mathrm{~A}$ [1]. A theoretical value of $550 \mathrm{~nm}$ for the insulating layer thickness with a leak current of $10^{9} \mathrm{~A}$ was defined by ProeTex (2007) [15].

Cardoen et al. [3] have shown that PES ribbons dip-coated with PI from DMF solutions at concentrations lower than $10 \mathrm{w} \%$ resulted in a homogeneous coating. However, those coatings did not provide sufficient insulation. Only a concentration of $10 \mathrm{w} \%$ and higher and coating speeds equal to or higher than $75 \mathrm{~mm} / \mathrm{min}$ led to fully insulating layers on the PES ribbons [3].

The solution that adheres to the substrate when withdrawn forms the film as the solvent evaporates [16]. From the LandauLevich equation, and underlined for dip-coating in the work of Arfsten et al., [17] it can be derived that the thickness of the layer can be tailored by varying the viscosity of the solvent (solution concentration) and the dip-coating withdrawal speed. Increasing either of these parameters will lead not only to a thicker dielectric layer but also to an increased risk of dielectric layer delamination. Previous research [2] confirmed this phenomenon that minimal speed at which an insulating coating is obtained decreases with increasing solution concentration.
It is obvious that there are still many problems to be solved in the deposition of polyimide by dip-coating as dielectric layer on a copper-coated PES filament with respect to the application in transistor architecture. Our present work concentrates on the morphology of the layer in relation to the process conditions as the choice of solvent, solution concentration, dip-coating speed and drying procedures. As demonstrated in this paper, our research resulted in a high-quality insulating polyimide layer with high homogeneity, minimum thickness and roughness.

\subsection{Materials}

Polyimide (PI) resin, purchased from Alfa Aesar, was dissolved in two different high-boiling point solvents (SigmaAldrich), namely 1-methyl-2-pyrrolidone (NMP) [10,18] and dimethylformaldehyde (DMF) $[19,20]$. Each solution was tested in a variety of concentrations.

A copper-coated polyester filament (PES) with a diameter of $888 \mu \mathrm{m}$ was dip-coated in one of these solutions to generate the polyimide dielectric layer on the filament's surface. The PES filament was purchased from Teijin, whereas the copper coating was applied on its surface as described by Van Genabet et al. [21].

Polyimide was chosen because it is known for its good flexibility [10], low conductivity, high breakdown voltage and high dielectric constant of approximately 3.5 in ambient conditions [10]. It is therefore ideal for application as dielectric material on textile substrates. Polyimide films are commercially available, for example, as Kapton Film (DuPont) [22] and are frequently used in the electronics industry. PI coatings result in durable and tough layers with excellent chemical stability and show good adhesion to various surfaces [23].

\subsection{Methods}

Solutions of polyimide in DMF $(7.5,10,12.5,15$ and $17.5 \mathrm{w} \%)$ and NMP (12.5 and $15 \mathrm{w} \%$ ) were prepared by slow magnetic stirring for $24 \mathrm{~h}$, inhibiting air bubble entrapment.

Before dip-coating in polyimide, the copper-coated PES filaments underwent a cleaning step. Alternatively, distilled water, oxidation with hydrogen peroxide, chloroform and dry methanol were tested as the cleaning agents to improve wettability and to remove impurities, dust, fat or other residues. The substrate was dipped into the respective solvent prior to the actual dip-coating process.

To avoid layer inhomogeneity and guarantee a constant thickness of the coated layer, a dip-coater located on a vibrationfree table in a clean room was used. Dip-coating is commonly used for depositing thin films on filaments or substrates with a three-dimensional shape.

The filaments were dip-coated in the solutions at a withdrawal speed between 5 and $150 \mathrm{~mm} / \mathrm{min}$. During dip-coating, the substrate is immersed into and withdrawn from the polyimide solution at a constant speed. 
Subsequent to dip-coating, the filament is dried at ambient conditions at $22^{\circ} \mathrm{C}$ or alternatively, at elevated temperatures $\left(60^{\circ} \mathrm{C}\right)$ for $24 \mathrm{~h}$ in vertical position, imitating continuous dipcoating conditions. Relative humidity during the dip-coating process itself depended on atmospheric conditions.

\subsection{Analysis}

After dip-coating, the layer was analysed with respect to its surface characteristics, thickness, electrical properties (resistance and leakage) and roughness.

\section{Surface Morphology}

Samples were visually examined with an optical microscope (Olympus), scanning electron microscope (SEM, Fei Quanta $200 \mathrm{~F}$, high vacuum mode, under high voltage of $20 \mathrm{kV}$ ) and a Focussed lon Beam (FIB) analyser.

\section{Thickness}

For measuring the thickness of the PI layer, the coated samples were embedded in historesin (Leica) to avoid compression of the layer when cutting it for cross-sectional inspection. Optical microscopy in combination with Cell^d software was used for defining the layer thickness on the filament's cross section.

\section{Electrical properties}

Testing the insulating properties regarding the leak current of the deposited layer was done on three levels with increasing accuracy, up to $\mathrm{pA}$.

\section{Resistance}

Initial testing of the insulating properties of the PI film was performed by measuring the resistance with a multimeter (digi tool IEC Digi 16). Two alligator clamps were attached each $10 \mathrm{~mm}$ from the border of the insulating layer. The distance between the clamps was $20 \mathrm{~mm}$ (Figure 2). Contacting was improved by using silver-based conductive adhesive (silver pigmented, Shieldokit 3980, Holland shielding systems). By using this intermediate layer, the risk of false measurements (bad contacts) could be eliminated and reliable measurements could be achieved. To get a first insight into the electrical properties of the dielectric layer, a minimal measured resistance of $200 \mathrm{M} \Omega$ was used as a threshold value for a sufficient level of insulation.

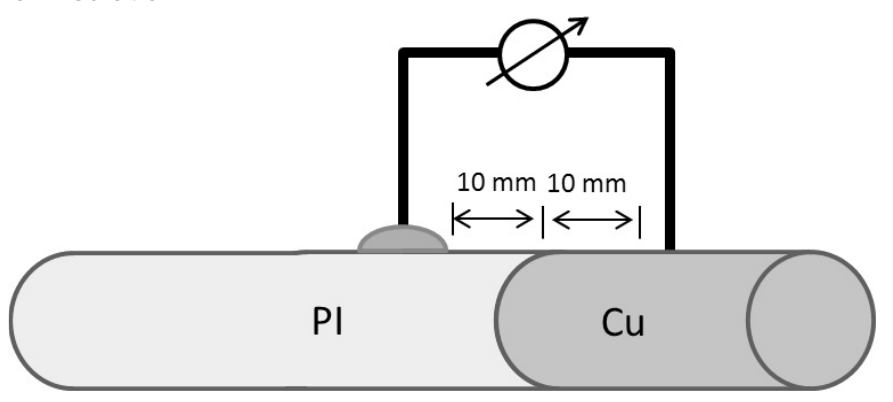

Figure 2. Initial determination of leak current through dielectric layer Leak current
In view of the final application as OFET, deposition of the contacts serving as source and drain electrodes is of importance with regard to dimensions. The contacts were applied by gold sputtering under vacuum. Using a shadow mask, the contacts could be limited to a length of $20 \mathrm{~mm}$ and approximately $240^{\circ}$ of the fibre's circumference, resulting in a surface of approximately $74 \mathrm{~mm}^{2}$. The contacts were applied in filament direction (longitudinal). The deposited gold electrodes ensured not only a much closer contact to the dielectric layer but also extend over a larger surface area compared with the previous testing with conductive adhesive. With the new contacts, the leak current was measured through the dielectric layer at an increasing voltage between the gate (copper-coated PES) and the gold contact. Initially, leak current was measured with an accuracy of $10^{5} \mathrm{~A}$ and $10^{4} \mathrm{~V}$ when applying a voltage of $60 \mathrm{~V}$. Following, the samples were tested at even higher accuracy (up to $\mathrm{pA}$ ) with a sensitive $\mathrm{V}$-A measuring set-up. Voltage was increased from 0 to $10 \mathrm{~V}$ in steps of $1 \mathrm{~V}$.

\section{Roughness}

To round-up the surface analysis, atomic force microscopy (AFM) in semi-contacted tapping mode was used to measure the roughness of the dielectric layer. Roughness values were calculated using WSXM 4.0 software. AFM plots show the number of events of an encountered height deviation in function of the value of that height deviation in $\mathrm{nm}$. To compare the relative roughness, the root mean square of this value was used.

\section{Results and Discussion}

\section{Pretreatment}

In general, dip-coating requires good wetting properties of the substrate's surface. Tests have been conducted on flat copper foil [3]. To test the effect of a variety of pretreatments, the surface was treated with different substances: distilled water, oxidation with hydrogen peroxide, chloroform and methanol. Both visual inspection and analysis under optical microscope showed that best results without damaging the copper layer could be achieved when using methanol. This pretreatment resulted in a decrease in the contact angle from $45^{\circ}$ to $20^{\circ}$ on cupper foil with solution from PI/DMF $7 \mathrm{w} \%$ [3]. In the following experiments, the samples were pretreated with methanol for 2 min before dip-coating to remove dirt particles and improve the contact angle. After that to avoid any water contamination, the samples were dipped into DMF and dried. The surface energy of the substrate can be influenced by absorption of liquids, too $[24,25]$. SEM analysis did not show any significant differences in morphology of the polyimide layer itself (see Figure 3). Hence, even though there is no visible effect on the layer, it is recommended to pretreat the fibres with methanol to remove dirt and other residues ensuring reproducibility and quality of the coating.

\section{Dip-coating}

To find the optimal process conditions, the solution PI/DMF as well as PI/NMP was prepared in a variety of concentrations. 

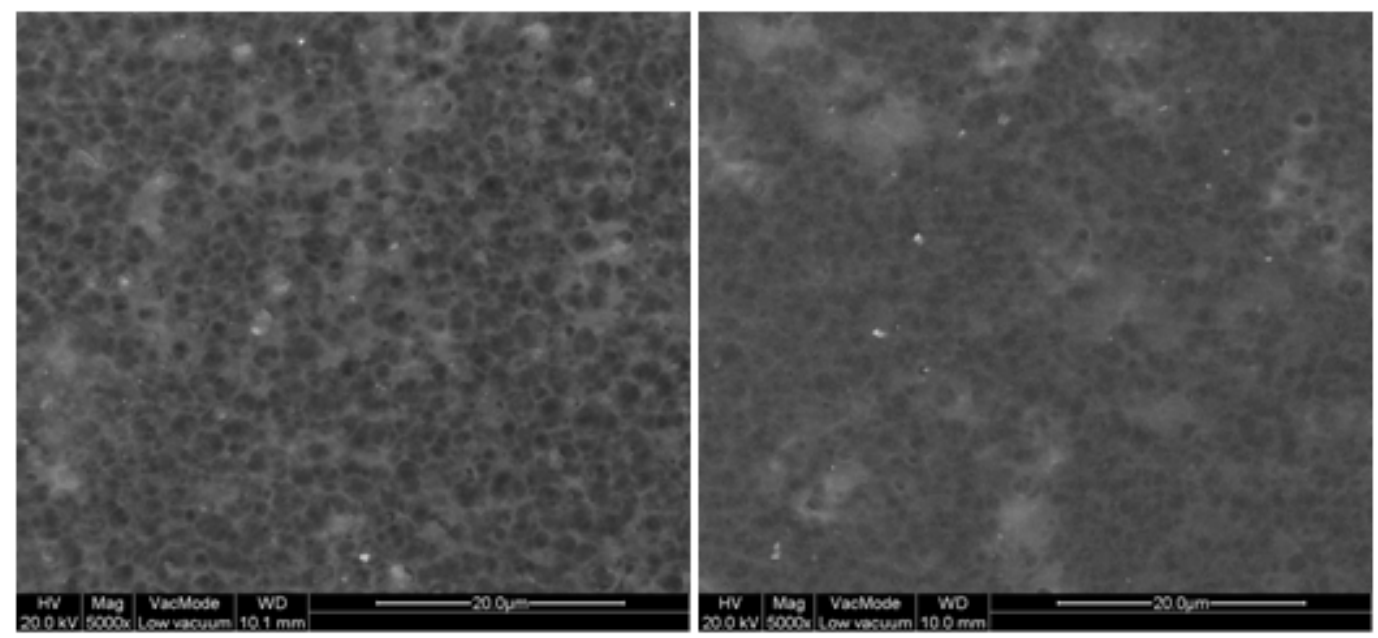

Figure 3. SEM images: DMF $15 \mathrm{w} \%$ dip-coated at $25 \mathrm{~mm} / \mathrm{min}$ without (left) and with methanol pretreatment (right)

In addition, the withdrawal speed varied during the dip-coating process.

With both solutions, a whitening of the deposited polyimide layer was observed within the first $60 \mathrm{~s}$ after withdrawal of the filament from the solution in ambient conditions (see Figures 4 and 5).

This effect was less obvious for withdrawal from solutions with lower concentration. In this case, the layer stayed almost transparent and only a slight change in colour could be recognized. At constant solution concentration, the higher the withdrawal speed, the more opaque the layer became. As stated beforehand, increasing withdrawal speed or solution concentration influenced the layer thickness. Therefore, we assumed that the layer thickness has an influence on the whitening of the layer.

Polyimide usually is transparent to the spectrum of visible light; it does not comprise light-absorbent additive molecules such as pigments or dyes. Anyway, diffuse reflection can occur caused by light rays that get reflected in many random directions on rough and irregular surfaces. The cavities that were detected through SEM microscopy are therefore the cause for a decrease in optical transmission. This means that the cavities (voids or cracks) reflect the light randomly, leading to diffuse reflection and, therefore, the opaque colour of the layer.
Cavities could act as charge traps in subsequent application as dielectric layer in the transistor architecture. Hence, the cavities trap mobile charges flowing at the interface to the semi-conductive layer. This reduces field effect mobility and, therefore, severely affects performance of the transistor. Consequently, it is important to reduce the layer's roughness for further research.

To further assess the surface morphology, a SEM at a magnification of $5000 x$ was used. SEM analysis showed that

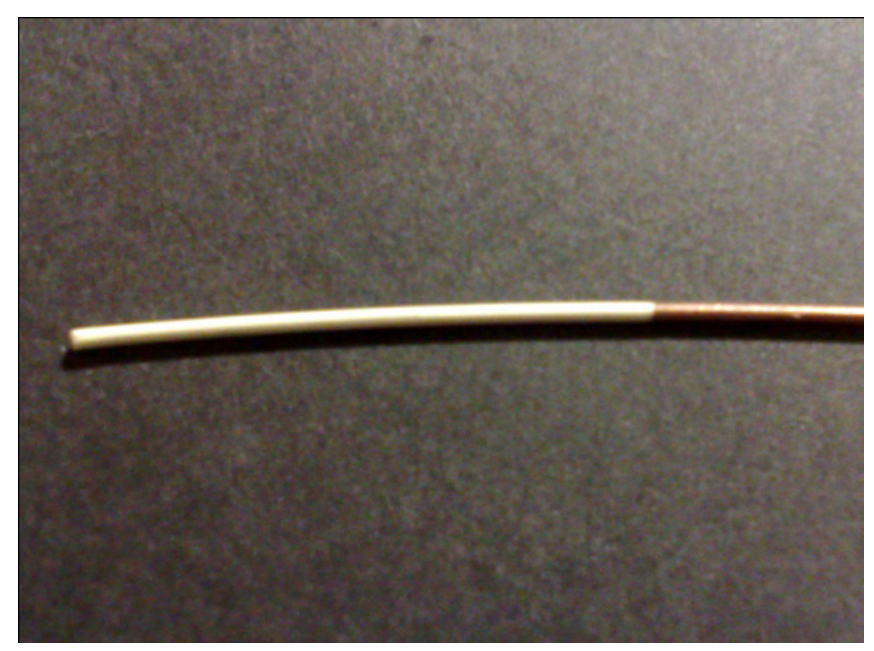

Figure 4. Whitening of polyimide layer on copper-coated polyester filament (PI/NMP 12.5w\%, $50 \mathrm{~mm} / \mathrm{min})$
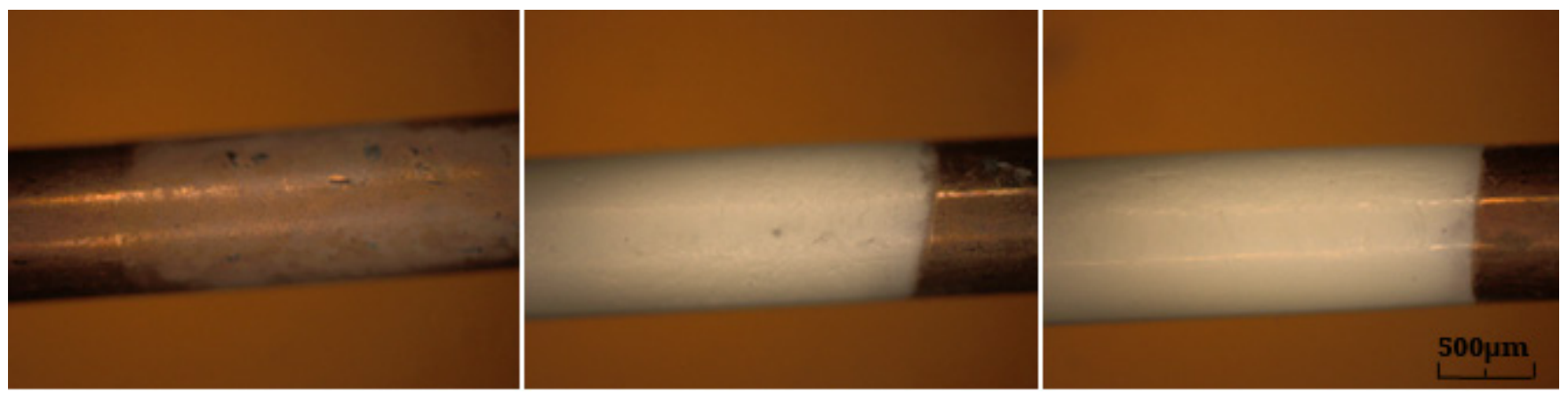

Figure 5. Copper-coated PES filament dip-coated in PI/DMF at $50 \mathrm{~mm} / \mathrm{min}$ with a PI concentration of $10 \mathrm{w} \%$, 15 and $17.5 \mathrm{w} \%$ (from left to right) 
small cellular holes in the order of magnitude of micrometres were formed at the layer surface. Increasing either the withdrawal speed (from 5 to $100 \mathrm{~mm} / \mathrm{min}$ ) or concentration (7.5-17.5w\%), leads to bigger cavities (Figures 6 and 7).

Several parameters were investigated to improve the dipcoated polyimide layer morphology. The ambient conditions in the clean room where the dip-coating took place were assessed and found to be unstable. A mobile weather station indicated a temperature of $22.0^{\circ} \mathrm{C}$ and a relative humidity of $25 \%$. Humidity was an eventual threat to the quality of the layer morphology. The possibility that relative humidity was responsible for the discoloration also explained why the samples that were transparent still had white ends at the bottom: the samples were drying in upright position. Once the filament got withdrawn from the solution, gravity causes the solution to flow downwards on the sample resulting in a thicker droplet at the lowest part of the fibre. Since this part of the layer is thicker than the rest, it requires a longer time to dry and gave the layer time to absorb water.
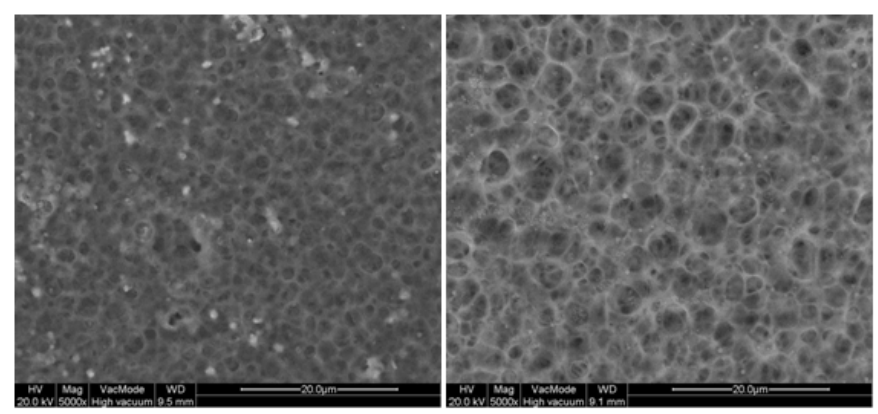

The environmental humidity was reduced from $25 \%$ to approximately $5 \%$ when drying the freshly coated samples in an oven at $60^{\circ} \mathrm{C}$. Drying the samples in the oven at low humidity led to complete transparency of the polyimide layer after drying. Samples were analysed at 5000× magnification with a SEM and compared with the samples dried at ambient conditions. The effect was impressive. Figure 8 shows SEM images of a PI/NMP-coated fibre drying in a relative humidity $(\mathrm{RH})$ of $30 \%, 18 \%$ and $5 \%$. The increased transparency of the layer is an indicator that the diffuse reflection was reduced, hence an improvement of the surface morphology of the layer. Low humidity drying led to a decrease in number and sizes of voids in the layer.

In literature, this phenomenon is described as vapourinduced phase separation $[18,26,27]$. NMP is a very good solvent for water, which acts at the same time as a nonsolvent for the polyimide molecules. In general, phase separation can be triggered by the so-called wet immersion processes. Here, the polymer solution is applied onto a

Figure 6. SEM images of PI dielectric layer dip-coated from 12.5w\% PI/DMF solution with respective withdrawal speed of 5, 25, 50 and $100 \mathrm{~mm} /$ min (from left to right)
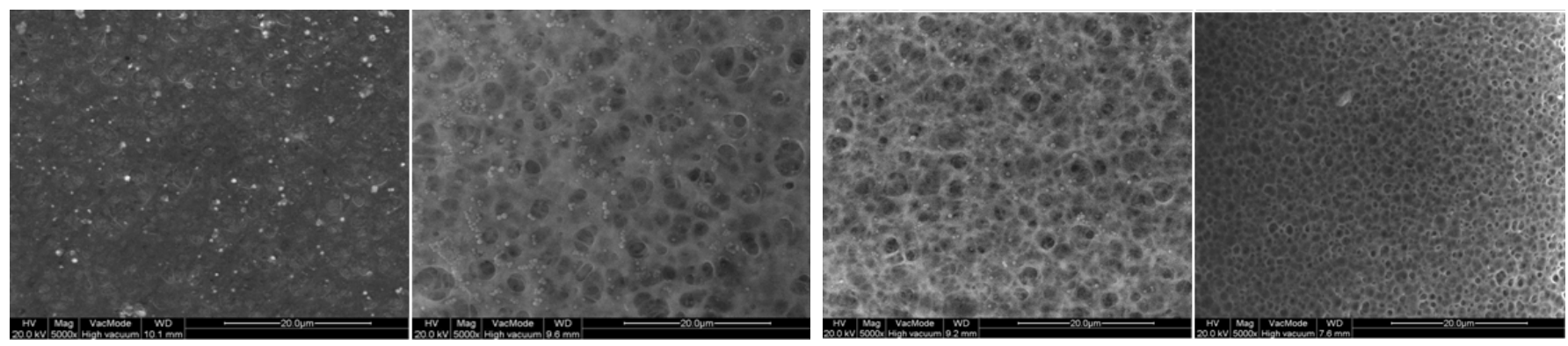

Figure 7. SEM images of PI dielectric layer dip-coated at a withdrawal rate of $50 \mathrm{~mm} / \mathrm{min}$ and PI/DMF solution concentration of $7.5 \mathrm{w} \%$, $12.5 \mathrm{w} \%$, $15 \mathrm{w} \%$ and $17.5 \mathrm{w} \%$ (from left to right)
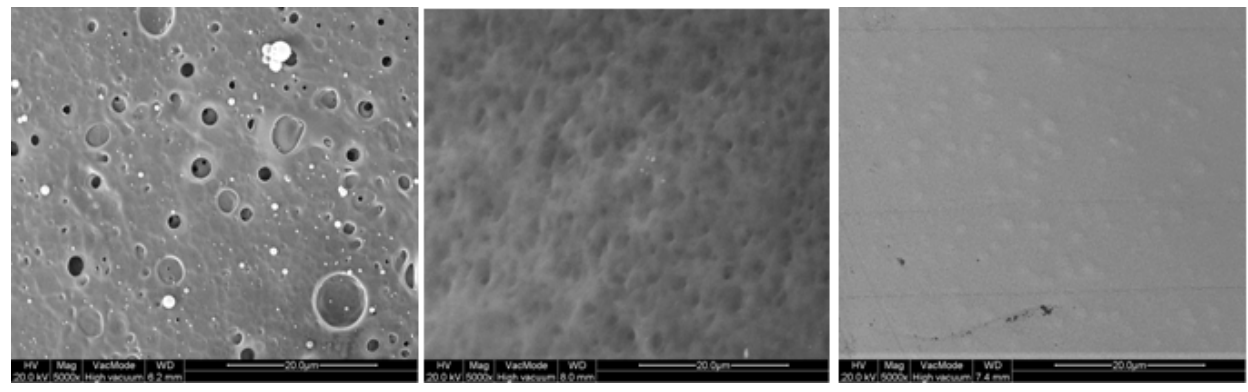

Figure 8. SEM images of samples dip-coated in $12.5 \mathrm{w} \% \mathrm{PI} / \mathrm{NMP}$ solution at $50 \mathrm{~mm} / \mathrm{min}$ at a relative humidity of $30 \%, 18 \%$ and $5 \%$ (from left to right).Dip-coating at $5 \%$ humidity shows the highest transparency. 
substrate and immerged into a coagulation bath. The mass exchange is very rapid upon extraction of the solvent and penetration of non-solvent solidification. When solvent extraction is too fast, this process often leads to the formation of large holes.

In 2010, Bouyer elaborated on the behaviour of polyimidesolvent-water systems. In situ measurements of the concentrations of $\mathrm{PEI} / \mathrm{NMP} /$ water system were conducted at a relative humidity of $75 \%$ during the drying process [26]. A reduction in the relative humidity from $75 \%$ to $43 \%$ at $40^{\circ} \mathrm{C}$ has led to an increase in the demixing time by a factor of 6 leading to a more uniform cell distribution along the membrane thickness. If the value of the relative humidity is even more reduced, a dense film would be formed rather than a porous membrane. The minimum value of relative humidity to induce demixing has been reported at $27 \%$, below this concentration dense polymer films were obtained from solutions with NMP $[26,27]$.

In PI, a similar mechanism as in PEI has been observed and the same solvent was used; consequently, the effect is believed to be similar. The obtained morphology was characterized by large cells near the air/solution interface and also a whitening of the layer was observed. According to Kim et al., a similar morphology is obtained when quenching a DMSO/PI system in a water coagulation bath at $8^{\circ} \mathrm{C}[18]$.

To compare this previous work, a cross section was made of a sample coated from $12.5 \mathrm{w} \% \mathrm{PI} / \mathrm{NMP}$ solution at speed of $50 \mathrm{~mm} / \mathrm{min}$ and analysed with a focused ion beam (FIB) microscopy. The sample dried at the ambient relative humidity of $30 \mathrm{w} \%$ and is shown in Figure 9. A large macroscopic-void structure could be observed.

When drying the coating at low relative humidity, small cavities seemed to have disappeared. Water begins to evaporate if the chemical potential of water in the liquid phase reaches its chemical potential in the gas phase. Depending on the respective evaporation rate, the composition path could stay in the homogeneous region [27]. For the PI/NMP system, this effect was stronger. This might be explained by the larger difference in the boiling point of NMP $\left(207^{\circ} \mathrm{C}\right)$ and water compared with the difference between DMF $\left(153^{\circ} \mathrm{C}\right)$ and water. The use of high boiling point solvents results in a better morphology in film deposition [28-30].

As for the samples dip-coated in DMF-based solution, it was observed that whitening of the polyimide film occurred already within $60 \mathrm{~s}$ after coating. Putting the samples immediately into the oven $\left(60^{\circ} \mathrm{C}\right)$ reversed the process and dense, transparent films were obtained. At lower dip-coating speed and, therefore, longer coating procedure, the whitening became irreversible even after drying in the oven. SEM analysis led to the conclusion that the PI/DMF solution is even more sensitive to humidity than the PI/NMP mixture. To start phase separation, in the case of PI/DMF, less water needs to be absorbed. All samples dip-coated from $\mathrm{PI} / \mathrm{NMP} 12 \mathrm{w} \%$ and dried at $60^{\circ} \mathrm{C}$ were transparent (see Figure 10).

Results from thickness measurements are summarized in Figures 11 and 12. The exact thickness values have been published beforehand.

In Figure 11, the thickness is measured at a respective relative humidity in the oven at $60^{\circ} \mathrm{C}$, at a relative humidity of $15 \%$ (transparent layers) and at a relative humidity of $30 \%$ (whitening of the film). It was observed that the thickness increased with increasing relative humidity. This effect became stronger as the withdrawal rate increased. The same was observed for samples coated from DMF solution at 15 and $17.5 \mathrm{w} \%$, as shown in Figure 12. Here, only two conditions were compared, dry conditions at $60^{\circ} \mathrm{C}$ and $5 \% \mathrm{RH}$ and in ambient temperature and $>15 \% \mathrm{RH}$. The increase in thickness was higher compared with the samples coated from NMP solution showing the effect of the increased sensitivity of DMF solutions towards humidity. Phase separation that occurs at high relative humidity levels

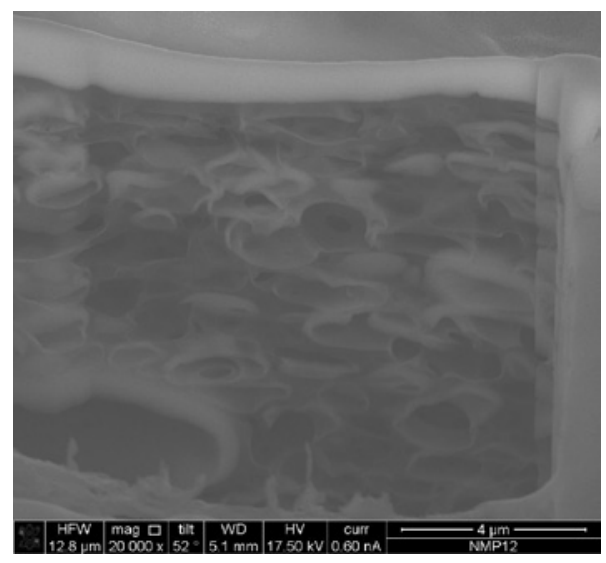

Figure 9. FIB cross section of the sample coated with a dielectric layer from $12.5 \mathrm{w} \% \mathrm{PI} / \mathrm{NMP}$ solution at $50 \mathrm{~mm} / \mathrm{min}$ in ambient conditions with $\mathrm{RH} 30 \%$.
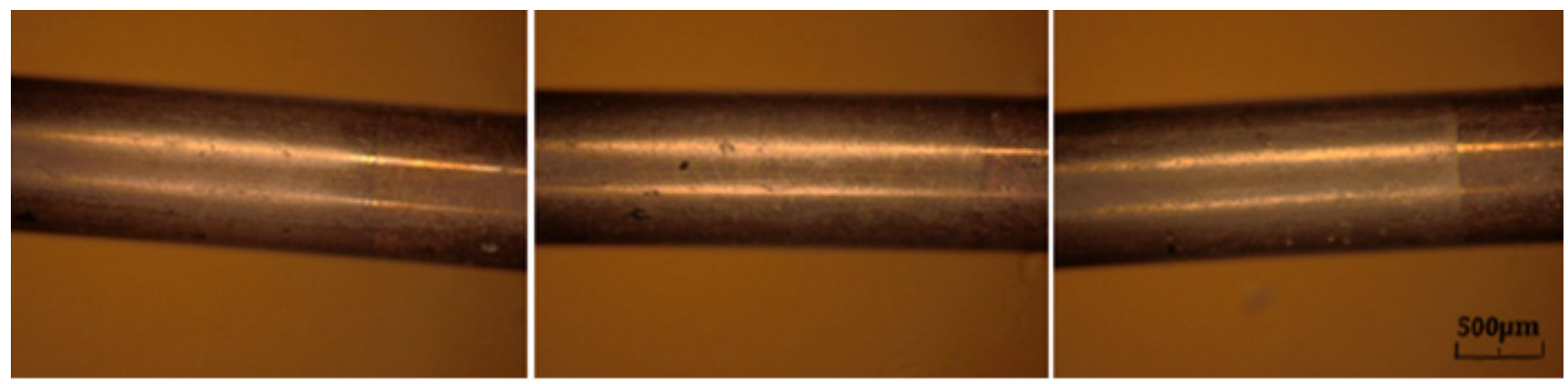

Figure 10. Copper-coated PES filaments dip-coated in PI/NMP solution at $12 \mathrm{w} \%$ concentration and at a withdrawal speed of $5,25 \mathrm{and} 75 \mathrm{~mm} / \mathrm{min}$, respectively. The layer remains transparent. 


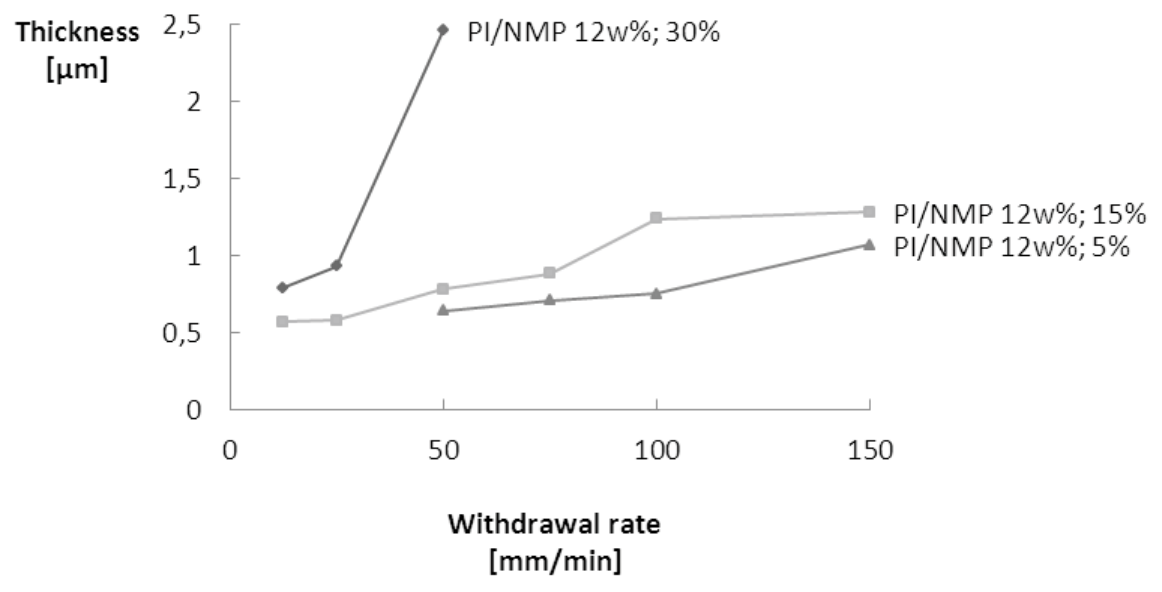

Figure 11. Thickness of PI layer coated from $12.5 \mathrm{w} \% \mathrm{PI} / \mathrm{NMP}$ solution in function of the withdrawal rate at different levels of relative humidity.

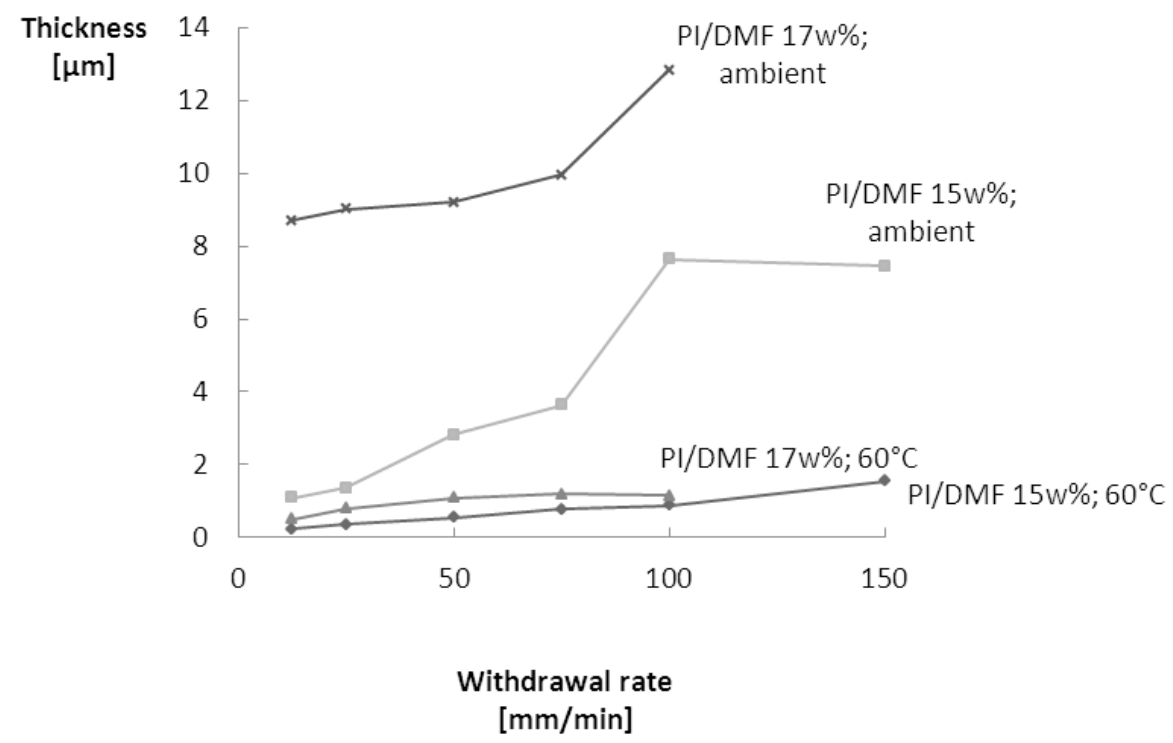

Figure 12. Thickness of PI layer coated from 15 and $17.5 \mathrm{w} \% \mathrm{PI} / \mathrm{DMF}$ solutions in function of the withdrawal rate at different levels of relative humidity.

result in a cellular morphology; consequently, the cavities formed in the layer do fill a much larger volume with the same amount of material deposited.

The overall layer thickness in dry conditions shows that for compatible speed and solvent concentration, the layer thickness is lower when NMP is used as the solvent. This effect became stronger as the withdrawal rate increased. At a withdrawal speed of $25 \mathrm{~mm} / \mathrm{min}$ and higher, all deposited layers were insulating. This corresponds with an approximate thickness of $600 \mathrm{~nm}$.

In this publication, we are referring to already published data for the insulating properties of the PI layer. When applying a silver paste as the contact medium during measuring insulating properties, a sufficient insulating layer $(R>200 \mathrm{M} \Omega$ ) was obtained when the dip-coating speed was $50 \mathrm{~mm} / \mathrm{min}$ at a concentration of $12.5 \mathrm{w} \%$. At a lower concentration, all samples remained conductive over the entire range of withdrawal speed. At higher concentrations, an insulating layer was achieved already at lower speed. At $15 \mathrm{w} \%$, a withdrawal speed of $10 \mathrm{~mm} /$ min resulted in an insulating layer. Thickness measurements lead to the conclusion that a threshold layer thickness of about $600 \mathrm{~nm}$ needs to be reached to be sufficiently insulating.

To assess the improvement in morphology detected by SEM analysis (see Figures 6-8), AFM scanning was chosen for the quantification of the results. A sample coated in $15 \mathrm{w} \%$ $\mathrm{PI} / \mathrm{DMF}$ at $12.5 \mathrm{~mm} / \mathrm{min}$ was compared with one coated in $12.5 \mathrm{w} \% \mathrm{PI} / \mathrm{NMP}$ at $50 \mathrm{~mm} / \mathrm{min}$ dried in ambient conditions. As a reference point for comparison, the roughness of the underlying copper layer was measured. Provided that the copper layer has a roughness of $45 \pm 1 \mathrm{~nm}$, the coating with DMF and NMP as a solvent showed a roughness of $107 \pm 2$ and $34 \pm 5 \mathrm{~nm}$, respectively. Voids that have been detected by SEM beforehand also were clearly visible through AFM imaging. Coatings using DMF led to dramatically increased roughness compared with the roughness of the underlying copper coating.

When comparing the roughness of the initial copper layer, the surface roughness got reduced when using NMP as a solvent for polyimide dip-coating. We concluded that dip-coating with 
NMP as solvent resulted in a better morphology than with DMF, even at higher withdrawal speeds. A roughness of $34 \mathrm{~nm}$ at the interface of dielectric and semiconductor is still considered very rough. Someya et al. reported a roughness of $0.2 \mathrm{~nm}$ of a spincoated PI layer on a poly(ethylene naphthalate) flat substrate [31]. Therefore, further research was necessary to improve the morphological properties of the layer with NMP as a solvent.

As a final comparison, samples coated from $15 \mathrm{w} \% \mathrm{PI} / \mathrm{NMP}$ as well as PI/DMF solution were produced at $50 \mathrm{~mm} / \mathrm{min}$ dipcoating speed and dried at $60^{\circ} \mathrm{C}$. Their roughnesses were assessed using AFM analysis (Figure 13). Both samples' surfaces looked perfectly smooth and the calculated roughness for both samples was almost equal with an average value of $0.375 \pm 0.007 \mathrm{~nm}$. This is an improvement with a factor of 285 compared with the first PI/DMF-coated samples, which were dried in high relative humidity. Consequently, the material's potential to be used as dielectric at the interface with the semiconductor without excessive charge entrapment has increased dramatically. Obtained roughness was comparable to the result obtained by Somaya et al., who reported a roughness of $0.2 \mathrm{~nm}$ rms of a PI layer spin-coated on a flat poly(ethylene naphthalate) substrate.

\section{Conclusions}

The polyimide layer was optimized in its properties and polyimide as dielectric material was assessed for application in fibrous transistors.

We showed that polyimide applied by dip-coating on a copper-coated polyester filament can serve as dielectric layer for a transistor architecture for application in smart textiles. DMF and NMP have been assessed as potential polyimide solvents and the dip-coating process has been optimized. Evaluation by optical microscopy, SEM and AFM has shown that the dielectric layer is sufficiently smooth to be used at the interface to the semiconductor. A roughness of $0.375 \pm 0.007 \mathrm{~nm}$ has been achieved by controlling humidity during the drying process after dip-coating, in combination with optimization of the $\mathrm{Pl} /$ solvent concentration and the dipcoating withdrawal speed. Using NMP as a solvent led to superior results.

The results presented enable us to proceed with the investigation of the subsequent layer of the transistor architecture, the organic semiconductor. This brings us one step closer to a working transistor on a filament for true integration into smart textiles.

\section{References}

[1] Maccioni, M., et al., Towards the textile transistor: Assembly and characterization of an organic field effect transistor with a cylindrical geometry. Applied Physics Letters, 2006. 89(14).

[2] Van Genabet, B., Synthesis and characterisation of copper, polyimide and TIPS-pentacene layers in the development of a solution processed fibrous transistor, in Master Thesis Universiteit Gent2010, Universiteit Gent: Gent.

[3] Cardoen, J., Ontwikkeling van transistor vezels, in Master Thesis Universiteit Gent2007.

[4] PlasticElectronics, SYSTEX D5 5 VISION PAPER 2011.

[5] Cherenack, K. and L. van Pieterson, Smart textiles: Challenges and opportunities. Journal of Applied Physics, 2012. 112(9).

[6] Marculescu, D., et al., Electronic textiles: A platform for pervasive computing. Proceedings of the leee, 2003. 91(12): p. 1995-2018.
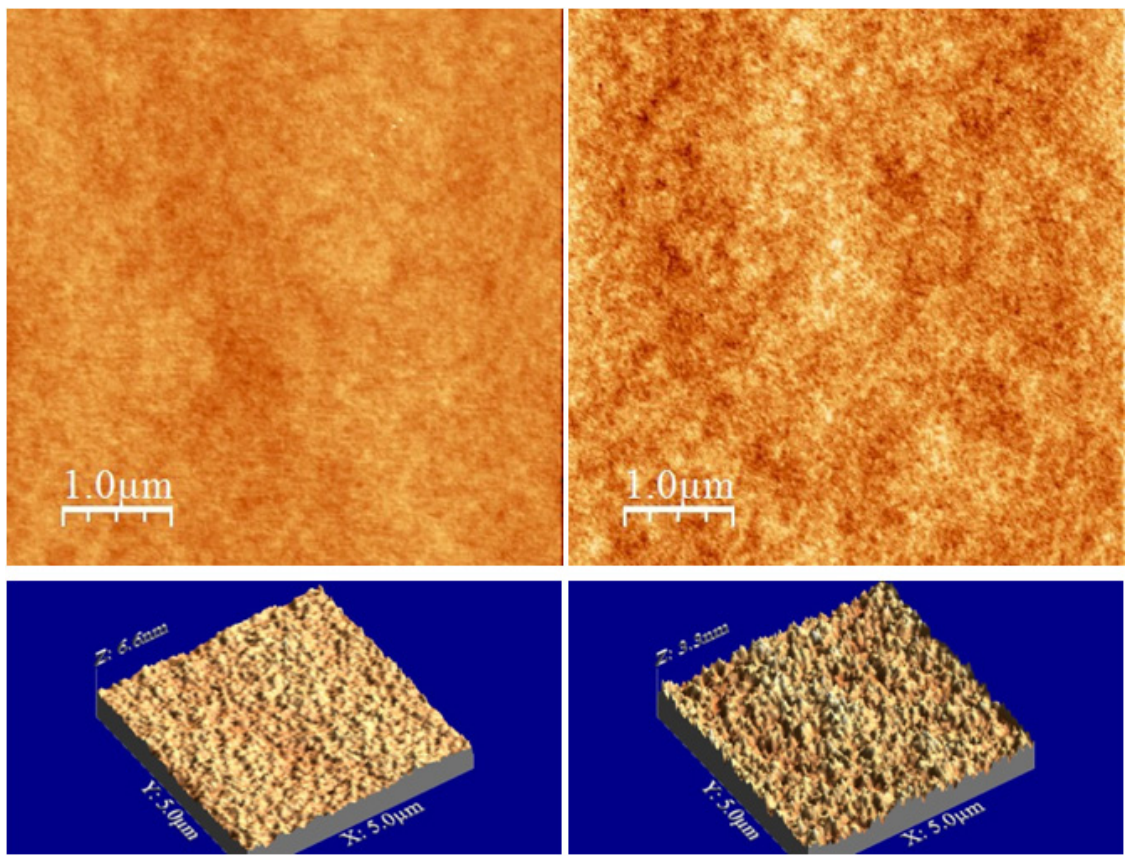

Figure 13. AFM images of samples coated with $15 \mathrm{w} \%$ solutions PI/DMF and PI/NMP, at a withdrawal rate of $50 \mathrm{~mm} / \mathrm{min}$ from DMF (left) or NMP (right). Their 3D representations of the surface are given (below). The surface looks perfectly smooth. 
[7] Marculescu, D., et al., Challenges and opportunities in electronic textiles modeling and optimization, in 39th Design Automation Conference, Proceedings 20022002. p. $175-180$.

[8] Wagner, H.D., E. Wiesel, and H.E. Gallis, SPREADING OF LIQUID DROPLETS ON CYLINDRICAL SURFACES - ACCURATE DETERMINATION OF CONTACT-ANGLE. Interfaces in Composites, ed. C.G. Pantano and E.J.H. Chen. Vol. 170. 1990. 141-145.

[9] Schwarz, A., et al., Steps Towards a Textile-Based Transistor: Development of the Gate and Insulating Layer. Textile Research Journal, 2010. 80(16): p. 1738-1746.

[10] Feili, D., et al., Flexible organic field effect transistors for biomedical microimplants using polyimide and parylene $C$ as substrate and insulator layers. Journal of Micromechanics and Microengineering, 2006. 16(8): $p$. 1555-1561.

[11] UGent, Why Textiles? - Benefits of Textiles. Smart Textiles Salon Vol.3, 2013. 3.

[12] Min, H.G., et al., Behavior of pentacene molecules deposited onto roughness-controlled polymer dielectrics films and its effect on FET performance. Synthetic Metals, 2013. 163: p. 7-12.

[13] Shi, W.W., et al., Progress of the improved mobilities of organic field-effect transistors based on dielectric surface modification. Acta Physica Sinica, 2012. 61(22).

[14] lazykov, M., et al., Atomic force microscopy analysis of morphology of thin pentacene films deposited on parylene-C and benzocyclobutene. Surface Science, 2013. 607: p. 170-173.

[15]PROETex, D8.1 Report on fibre design for different electronic functions (transistors, sensors), 2007.

[16] Bormashenko, E., et al., Mesoscopic and submicroscopic patterning in thin polymer films: Impact of the solvent. Materials Letters, 2005. 59(19-20): p. 2461-2464.

[17] Arfsten, N.J., et al., Investigations on the angle-dependent dip coating technique (ADDC) for the production of optical filters. Journal of Sol-Gel Science and Technology, 1997. 8(1-3): p. 1099-1104.

[18] Kim, J.H., et al., Phase behavior and mechanism of membrane formation for polyimide/DMSO/water system. Journal of Membrane Science, 2001. 187(1-2): p. 47-55.

[19]Guo, M.C. and X.G. Wang, SYNTHESIS AND CHARACTERIZATION OF POLYIMIDE WITH MAIN-
CHAIN PHOTOSENSITIVE GROUPS AND HYDROXYL SIDE-GROUPS. Acta Polymerica Sinica, 2008(11): $p$. 1113-1117.

[20] Ren, H.F., et al., Polyimide containing isosorbide units: Synthesis and characterization. Acta Polymerica Sinica, 2006(2): p. 248-252.

[21] Van Genabet, B., et al., Synthesis and characterization of copper, polyimide and TIPS-pentacene layers for the development of a solution processed fibrous transistor. Aip Advances, 2011. 1(4).

[22] DuPont. Kapton® polyimide film. 2012 [cited 201319.07. - 14:31]; Available from: http://www2.dupont.com/Kapton/ en_US/.

[23] AlfaAesar, Product Bulletin, Stock \#43656. 2013: p. 1.

[24] Schwarz, A., Analysis of wetting behaviour of an inclined fibre, 2005, Kaunas University of Technology: Kaunas.

[25] Warmoeskerken, M., Advanced and specialised textile processing, 2008, Univeristy of Twente: Twente, NL.

[26] Bouyer, D., et al., Morphological properties of membranes fabricated by VIPS process using PEI/NMP/water system: SEM analysis and mass transfer modelling. Journal of Membrane Science, 2010. 349(1-2): p. 97-112.

[27] Menut, $P$., et al., Structure formation of poly (ether-imide) films using non-solvent vapor induced phase separation: relationship between mass transfer and relative humidity. Desalination, 2002. 145(1-3): p. 11-16.

[28] Yong-Hoon, $K$., et al., Influence of solvent on the film morphology, crystallinity and electrical characteristics of triisopropylsilyl pentacene OTFTs. Journal of the Electrochemical Society, 2007. 154(12): p. H995-H998.

[29] Kim, J., et al., All-solution-processed bottom-gate organic thin-film transistor with improved subthreshold behaviour using functionalized pentacene active layer. Journal of Physics D-Applied Physics, 2009. 42(11).

[30] Choi, M.H., et al., Effect of active layer thickness on environmental stability of printed thin-film transistor. Organic Electronics, 2009. 10(3): p. 421-425.

[31] Someya, T., et al., A large-area, flexible pressure sensor matrix with organic field-effect transistors for artificial skin applications. Proceedings of the National Academy of Sciences of the United States of America, 2004. 101(27): p. 9966-9970. 